\title{
Planetary radio astronomy: Earth, giant planets, and beyond
}

\author{
H. O. Rucker ${ }^{1,2}$, M. Panchenko ${ }^{1}$, and C. Weber ${ }^{1}$ \\ ${ }^{1}$ Space Research Institute, Austrian Academy of Sciences, Schmiedlstraße 6, 8042 Graz, Austria \\ ${ }^{2}$ Commission for Astronomy, Austrian Academy of Sciences, Schmiedlstraße 6, 8042 Graz, Austria
}

Correspondence to: H. O. Rucker (rucker@oeaw.ac.at)

Received: 20 January 2014 - Revised: 13 June 2014 - Accepted: 23 June 2014 - Published: 10 November 2014

\begin{abstract}
The magnetospheric phenomenon of non-thermal radio emission is known since the serendipitous discovery of Jupiter as radio planet in 1955, opening the new field of "Planetary Radio Astronomy". Continuous ground-based observations and, in particular, space-borne measurements have meanwhile produced a comprehensive picture of a fascinating research area. Space missions as the Voyagers to the Giant Planets, specifically Voyager 2 further to Uranus and Neptune, Galileo orbiting Jupiter, and now Cassini in orbit around Saturn since July 2004, provide a huge amount of radio data, well embedded in other experiments monitoring space plasmas and magnetic fields. The present paper as a condensation of a presentation at the Kleinheubacher Tagung 2013 in honour of the 100th anniversary of Prof. Karl Rawer, provides an introduction into the generation mechanism of non-thermal planetary radio waves and highlights some new features of planetary radio emission detected in the recent past.
\end{abstract}

As one of the most sophisticated spacecraft, Cassini, now in space for more than 16 years and still in excellent health, enabled for the first time a seasonal overview of the magnetospheric variations and their implications for the generation of radio emission. Presently most puzzling is the seasonally variable rotational modulation of Saturn kilometric radio emission (SKR) as seen by Cassini, compared with early Voyager observations.

The cyclotron maser instability is the fundamental mechanism under which generation and sufficient amplification of non-thermal radio emission is most likely. Considering these physical processes, further theoretical investigations have been started to investigate the conditions and possibilities of non-thermal radio emission from exoplanets, from potential radio planets in extrasolar systems.

\section{Introduction}

After the discovery of Jupiter as radio emitter (Burke and Franklin, 1955) planetary radio emission was established as an essential part of magnetospheric physics, in particular since the observation of terrestrial auroral radio emission (Benediktov et al., 1966) and the detection of non-thermal radiation at the giant icy planets by Voyager 1 and 2 . The most intense planetary radio emission is auroral emission, observed close to the electron cyclotron frequency depending on the local magnetic field strength. The radio generation mechanism is the electron cyclotron maser instability (CMI) and the source location is at high auroral regions or, in the case of the Jovian Io dependent radio component, in the Io flux tube at high latitudes.

Essential radio emission parameters of the known radio planets of our solar system are summarized in $\mathrm{Ta}$ ble 1 (after Zarka, 1998). The names of the respective radio components represent the corresponding wave length of the radio emissions. Note that for Uranus the values $<100 / 400 / 850 \mathrm{kHz}$ correspond to the minimum, peak and maximum frequency values of the Bsmooth component of Uranus kilometric radiation (UKR), $60 / 400 / 750 \mathrm{kHz}$ to the B-bursts, $15 / 60 / 120 \mathrm{kHz}$ to the $\mathrm{N}$ bursts and $100 / 150 / 300 \mathrm{kHz}$ to the dayside component. In the case of Neptune $\leq 20 / 350 / 600 \mathrm{kHz}$ corresponds to the smooth component, $550 /-/ \geq 1326 \mathrm{kHz}$ to the main bursty and $450 /-1520 \mathrm{kHz}$ to the anomalous bursty component.

In Sect. 2 this review briefly addresses the generation of nonthermal radio emission. By both STEREO-A and -B spacecraft not only an unambiguous determination of different radio components is possible, but the very faint radio signatures of a new component of decametric radiation of Jupiter have recently been detected. This new component with a modulation slighty slower (approx. $10 \mathrm{~min}$ ) than the 
Table 1. Summary of essential radio emission parameters of the solar system radio planets (after Zarka, 1998).

\begin{tabular}{|c|c|c|c|c|c|}
\hline & Earth & Jupiter & Saturn & Uranus & Neptune \\
\hline Rotation period & $24 \mathrm{~h}$ & $9 \mathrm{~h} 55.5 \mathrm{~m}$ & 10 h $39.4 \mathrm{~m}$ & $17 \mathrm{~h} 14.4 \mathrm{~m}$ & $16 \mathrm{~h} 6.5 \mathrm{~m}$ \\
\hline $\begin{array}{l}\text { Generic radio } \\
\text { emission name }\end{array}$ & TKR (or AKR) & $\begin{array}{l}\mathrm{bKOM} / \mathrm{HOM} / \\
\text { DAM }\end{array}$ & SKR & UKR & NKR \\
\hline $\begin{array}{l}\text { Spectrum } \\
\text { (minimum/ } \\
\text { peak/ } \\
\text { maximum), } \\
\text { kHz }\end{array}$ & $\begin{array}{l}\sim 30 \\
\sim 250 \\
\sim 800\end{array}$ & $\begin{array}{l}\text { for DAM: } \\
? \\
15000 \\
40000\end{array}$ & $\begin{array}{l}\leq 20 \\
100-400 \\
1200\end{array}$ & $\begin{array}{l}<100 / 400 / 850 \\
60 / 400 / 750 \\
15 / 60 / 120 \\
100 / 150 / 300\end{array}$ & $\begin{array}{l}\leq 20 / 350 / 600 \\
550 /-/ \geq 1326 \\
450 /-/ 520\end{array}$ \\
\hline $\begin{array}{l}\text { Flux density } \\
\text { at } 1 \mathrm{AU}, \\
\mathrm{W} \mathrm{m}^{-2} \mathrm{~Hz}^{-1}\end{array}$ & $\begin{array}{l}5 \times 10^{-21} \text { up to } \\
10^{-19}\end{array}$ & $\begin{array}{l}10^{-19} \\
10^{-17}\end{array}$ up to & $2 \times 10^{-20}$ & $\sim 4 \times 10^{-22}$ & $\begin{array}{l}1-7 \times 10^{-22} \\
3 \times 10^{-21}\end{array}$ \\
\hline
\end{tabular}

System III Jupiter rotation appears to have a source location somewhere in the Io torus, unlike the other Jovian radio components, which are located either in the Jovian auroral regions or in the Io flux tube close to Jupiter (Sect. 3). The intriguing observations by Cassini reveal a series of fascinating phenomena, including the variable rotational modulation of the Saturn Kilometric Radiation (SKR) (Sect. 4). In Sect. 5 this review on specific highlights concludes with a first glimpse into potential radio emission from exoplanets and corresponding theoretical investigations.

\section{Generation of non-thermal radio emission}

CMI is a wave-particle-interaction process which is based on a resonant exchange of angular momentum between energetic electrons precipitating along the auroral magnetic field lines and an electromagnetic wave. Weakly relativistic beams of electrons (few $\mathrm{keV}$ ) are the responsible agents for the generation of non-thermal radio emission. In plasmadepleted regions close to respective magnetic planet the velocity distribution of the beam electrons becomes instable due to collisions with upper atmospheric constituents. The converging magnetic field structure in the auroral regions reflects the electron population, now out of equilibrium, and in course of their gyromotion with the electron cyclotron frequency the electrons get in wave-particle resonance $\omega_{\mathrm{r}}-\frac{\omega_{\mathrm{ce}}}{\gamma}-k_{\|} v_{\|}=0$, with $\omega_{\mathrm{r}}$ as the radiation frequency, the electron cyclotron frequency $\omega_{\mathrm{ce}}$, the relativistic Lorentz term $\gamma$ and the Doppler shift $k_{\|} v_{\|}$parallel to the ambient magnetic field. This resonance condition is part of the dispersion relation (top equation in Fig. 1) (Wu and Lee, 1979; Wu, 1985) which provides optimum wave amplification when the resonance curve, i.e. the resonance ellipse in phase space, is best positioned in the loss cone (Louarn et al., 1990) (indicated by vertical arrows in Fig. 1). Thus the optimum conditions for wave amplification (plasma-depleted region with plasma frequency $\omega_{\mathrm{p}} \ll \omega_{\mathrm{ce}}, k_{\|} v_{\|}$small with regard to the other terms in the resonance condition) are met when the curve of integration along the resonance ellipse is mainly located in areas of $\frac{\partial F_{\mathrm{e}}}{\partial v_{1}}>0$, where $F_{\mathrm{e}}$ is the velocity distribution function of electrons. This so-called cyclotron maser instability (CMI) mechanism, leading to radiation generated by either loss cone distributions or shell distributions (Ergun et al., 2000) of electrons, is a direct generation mechanism of non-thermal radiation and ubiquitous in planetary auroral regions. The CMI mechanism produces radio waves with wave vectors almost perpendicular to the local magnetic field. In the case of Earth, the corresponding source regions can be sampled in situ by satellites (Ergun et al., 1998). The CMI theory predicts domination of the right-hand polarized extraordinary mode of the emission with frequencies close to the local electron's gyrofrequency. An extensive overview of the electron-cyclotron maser can be found in Treumann (2006).

\section{Jupiter radio emission}

Jupiter with the largest planetary magnetosphere in the solar system is a complex source of a powerful coherent nonthermal radio emission in a wide frequency range (Carr et al., 1983). Decametric radio emission (DAM), the strongest component of Jovian auroral radiation discovered by Burke and Franklin (1955) is observed in a form of arc shaped radio bursts (on a minute timescale) in a frequency range from few MHz up to $40 \mathrm{MHz}$ (Carr et al., 1983; Zarka, 1998). Depending on the time scales, the Jovian DAM exhibits different complex spectral structures. The S-bursts of DAM are detected as very short (milliseconds) impulsive spikes with fast drift in the time-frequency plane (Riihimaa, 1970, 1977; Leblanc et al., 1980). The occurrence of the S-bursts observation depends on the orbital position of the satellite Io. The S-bursts are observed as single events or as groups of S-burst trains. The "long" L-burst emission (e.g. Io and non-Io controlled DAM arcs) varies on the time scale of a few seconds. 


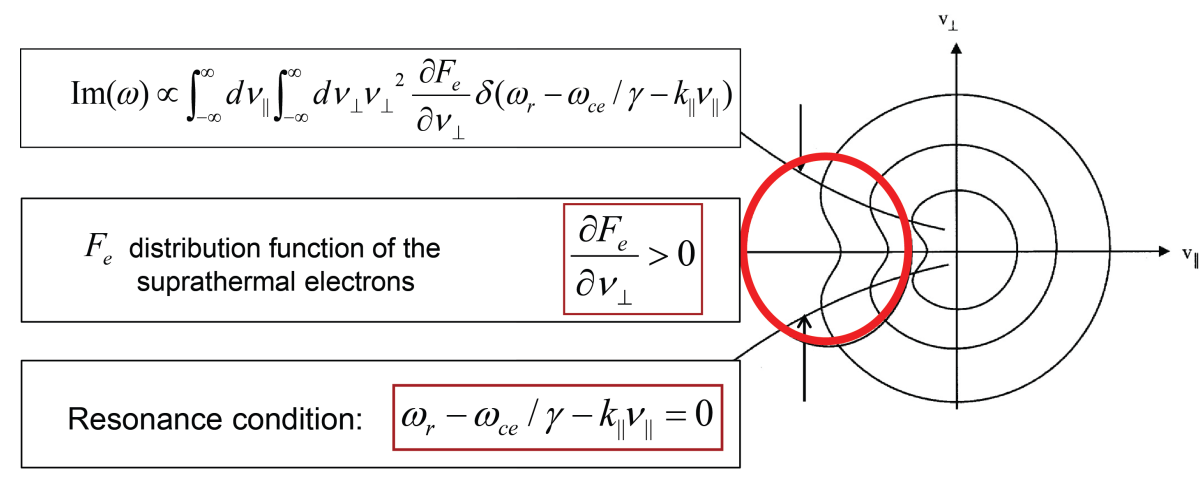

Figure 1. Growth rate of the dispersion relation of a radio wave (top equation) generated by an electron population with loss cone distribution with positive gradient of distribution function $F_{\mathrm{e}}$ (middle equation). The resonance condition (bottom equation) considering relativistic effects forms an ellipse in phase space.

Two types of the L-burst of DAM are observed: Io controlled "Io-DAM" and Io independent "non-Io DAM". The sources of the Io-DAM are located near the atmospheric footpoint of the Io flux tube. The electrodynamic interaction between Jupiter and its moon Io results in the acceleration and the precipitation of electrons into Jupiter's atmosphere and thus radiation of electromagnetic emissions in a frequency range from $1-2 \mathrm{MHz}$ up to $40 \mathrm{MHz}$. The observation probability of the Io-DAM strongly depends on the orbital phase of Io and Jovian Central Meridian Longitude (CML III) relative to the observer (Carr et al., 1983). The non-Io DAM is driven by the precipitating electrons accelerated by fieldaligned currents caused, most probably, by the breakdown of rigid corotation of the magnetosphere or by reconnection in the magnetotail and at the magnetopause (Saur et al., 2004).

In the case of Jupiter decametric radio waves are emitted in a hollow cone, where the radio emission is within the thin walls of the emission cone. The thickness of this cone mantle has been determined by Cassini and Wind stereoscopic observations (Kaiser et al., 2000) and recently been confirmed by Zörweg (2011) with STEREO-data, with cone mantle thickness in the order of about $1^{\circ}$.

DAM exhibits strong periodicities related to the rotation of the non-axisymmetric Jupiter's magnetosphere $(9.925 \mathrm{~h}$, System III) and Io orbital period (42.46 h) (Carr et al., 1983; Kaiser, 1993). The Io controlled component of DAM (IoDAM) is well organized into longitudinal systems related with the Io orbital position. The non-Io component of the DAM is a highly variable and sporadic radio emission, generally modulated by the rotation of the Jovian magnetosphere. Recently, Panchenko et al. (2010, 2013) and Panchenko and Rucker (2011) reported on the finding of new types of intense non-Io DAM bursts recorded by STEREO/WAVES, Wind/Waves and Cassini/RPWS space borne radio instruments in a frequency range from $5 \mathrm{MHz}$ to $12-16 \mathrm{MHz}$, which are rarely observable on the Earth due to ionospheric cut-off below $10 \mathrm{MHz}$. The examples of these bursts are presented in Fig. 2. These bursts were observed as series of arc- like radio features with negative time-frequency drift which reoccur with a surprisingly new averaged period of $10.07 \mathrm{~h}$ or $\sim 1.5 \%$ longer than the rotation rate of the Jovian magnetosphere and $\sim 1.5 \%$ shorter than the averaged periodicity in the Io plasma torus defined by System IV, $\sim 10.224 \mathrm{~h}$ (Sandel and Dessler, 1988). There is a preferable sector of Jovian CML III between $300^{\circ}$ and $60^{\circ}$, where the probability of observing the periodic bursts was found to be significant. No correlation with Jupiter's moons has been found.

The stereoscopic simultaneous observations from two spacecraft have shown that the radio sources of the periodic bursts corotate with Jupiter and, contrary to the non-Io DAM, their sources may be active during relatively longer periods of time (from 3 up to 7 Jupiter rotations), producing practically identical radio features. Moreover, the emission beam pattern of the periodic bursts has a strong anisotropy, because only one burst is generally observed per Jupiter rotation.

The measured solar wind parameters, ballistically propagated to the position of Jupiter, revealed a strong correlation between occurrence probability of the periodic bursts and the enhancement of the solar wind ram pressure. Additionally, the Lomb-Scargle spectral analysis has shown that the periodic bursts exhibit a tendency to occur in groups every $\sim 25$ days, i.e. the synoptic solar rotation period as viewed from Jupiter (Panchenko et al., 2013). The recent simultaneous observations of the periodic non-Io DAM using the radio experiment onboard the two STEREO spacecraft and the ground-based radio telescope URAN-2 (Poltava, Ukraine) have shown that the periodic bursts are observed as right and left hand polarized radio emission associated with the Northern and Southern magnetic hemispheres of Jupiter, respectively.

One possible source candidate for the energetic particles which may produce the periodic bursts of DAM is the Io torus. Steffl et al. (2006) have reported the finding of $10.07 \mathrm{~h}$ variation of ion azimuthal composition in the Io plasma torus observed by Cassini/UVIS. This is in very good agreement with the averaged period of the reoccurrence of the non-Io 

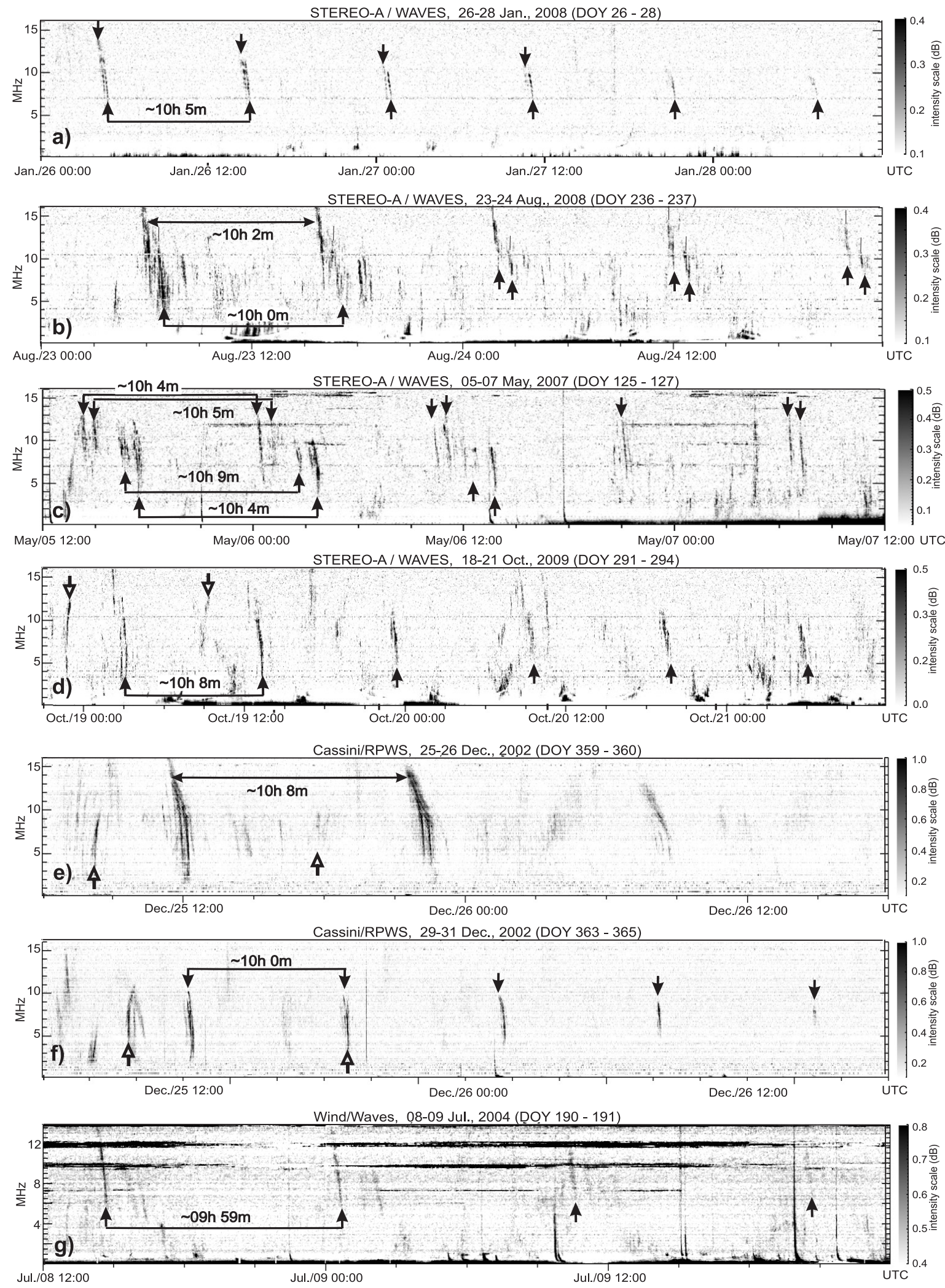

Figure 2. Examples of the periodic bursts observed by STEREO/WAVES, Cassini/RPWS and Wind/WAVES. The periodic bursts are marked by arrows. The average period of the burst repetition in each group is given. (From Panchenko et al., 2013) 


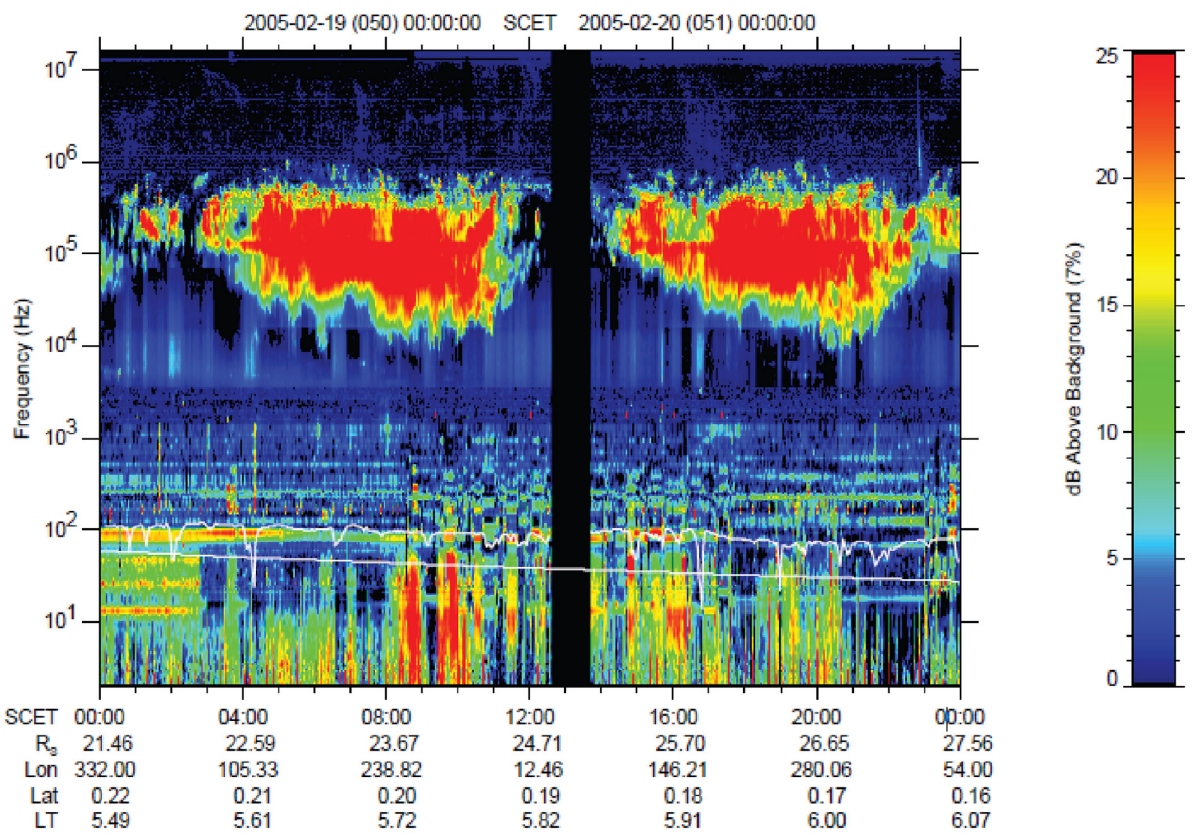

Figure 3. Dynamical spectrum of RPWS measurements of Cassini orbiting Saturn close to the equator. More than 2 Saturn rotations are performed in the $24 \mathrm{~h}$ time span. Patches of modulated SKR emission are seen in the frequency range of a few tens up to several hundreds of $\mathrm{kHz}$ (vertical axis) with radio flux densities more than $20 \mathrm{~dB}$ above background.

DAM periodic bursts. Furthermore, several episodes have been found when periodic non-Io DAM was observed simultaneously with so-called "bullseyes", U-shaped narrowband emissions in the low frequency $20-50 \mathrm{kHz}$ band (Kaiser and MacDowall, 1998), observed by Ulysses/URAP spacecraft. Farrell et al. (2004) explained the "bullseyes" emission as Jovian narrowband kilometric emissions (nKOM) generated in the radially extended plasma fingers developed in the course of the interchange instability in the Io torus. These latitudinal extended fingers may be a plasma source which supplies the cyclotron maser in the auroral region. Therefore, it has been suggested that the interchange instability in the Io torus, triggered by the solar wind pulses, may be a possible generation mechanism of the periodic non-Io DAM bursts (Panchenko et al., 2013).

Besides the main group of periodic bursts, two other groups of periodic features, rarely observed in the radio spectra, were found. There are "vertex-early" periodic non-Io DAM bursts or arcs with positive frequency drift and nonarc periodic radio features. In contrast to the main group, the "vertex-early" bursts reoccurred with a period close to the Jupiter System III rotation and typically were observed during 7-10 Jupiter rotations. The rarely recorded non-arc periodic radio features are observed as broad beamed radio emission lacking clear discrete features.

\section{Saturn radio emission periodicity}

The Voyager 1 and 2 flybys at Saturn enabled the detection (Kaiser et al., 1980) and further detailed investigations on the Saturn Kilometric Radiation (Kaiser et al., 1981; Kaiser and Desch, 1982). This radio emission is strongly modulated by the planetary rotation as Voyager measurements determined the period to $10.6 \mathrm{~h}$ (Desch, 1982). Radio measurements on the Ulysses spacecraft surprisingly showed that this periodicity apparently exhibits long-term variations by approximately one percent (Galopeau and Lecacheux, 2000). As pointed out by Gurnett et al. (2009), this small period variation should not be feasible for a planet rotating as fast as Saturn. However, this was confirmed by Cassini Radio and Plasma Wave Science (RPWS) measurements from 2002 to 2004 (Gurnett et al., 2005) with a new periodicity of $10.76 \mathrm{~h}$. This puzzle became even more complicated by the fact that further RPWS data analysis (Kurth et al., 2008) discovered a "second" component at the previously determined period of $10.6 \mathrm{~h}$.

Figure 3 shows a typical dynamic spectrum (radio flux density as a function of frequency and time) as observed by Cassini RPWS in orbit around Saturn, close to the equator. Here the rotational modulation is clearly visible in the kilometric wavelength range, corresponding to frequencies of several tens to hundreds of $\mathrm{kHz}$. Apart from studies on the average and statistical properties of the SKR based on the extensive Cassini RPWS measurements (Lamy et al., 2008), the variability of SKR periodicities has been the subject of 


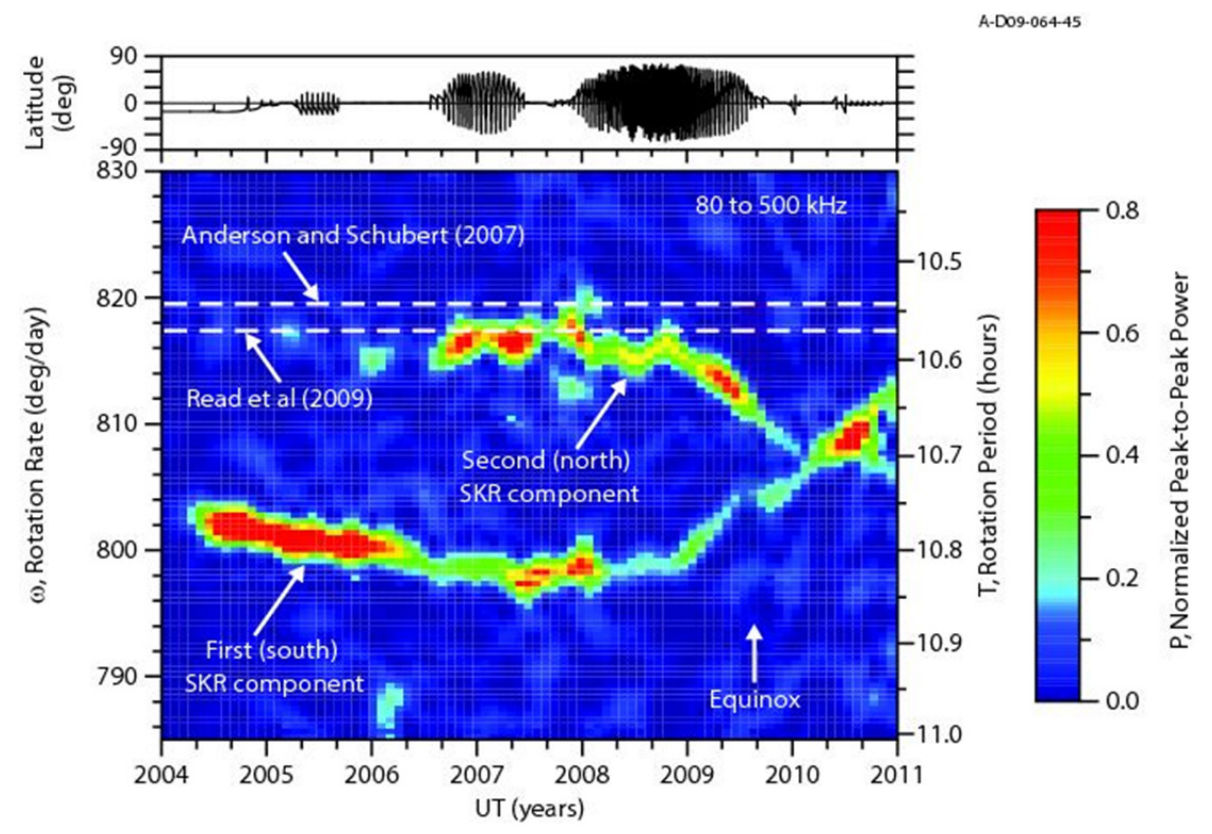

Figure 4. Normalized peak-to-peak power of SKR modulation in a diagram of rotation rate versus time. The "second SKR component" (Kurth et al., 2008) varies between 814 and $818 \%$ day and is close to rotation rates determined by Read et al. (2009) and Anderson and Schubert (2007). Note the crossing of the first (south) and second (north) SKR component several months after equinox.

a series of detailed investigations (Cecconi and Zarka, 2005; Gurnett et al., 2010; Lamy, 2011). The most intriguing fact is the appearance of a second component of SKR observed in the northern hemisphere of Saturn with a significantly different rotational periodicity (Fig. 4). This periodicity is close to the internal rotation rate of Saturn from gravity measurements considering Saturn's oblateness (Anderson and Schubert, 2007) and derived from atmospheric planetary-wave configurations (Read et al., 2009). A further surprising fact is the merging of the northern and southern rotation rates of both SKR components several months after equinox (several months delay has been observed, Fig. 4).

As has been discussed in Gurnett et al. (2009) the component observed by Cassini during the part of the orbit at northern latitudes $\left(>10^{\circ}\right)$ shows a rotation rate of $\sim 816^{\circ}$ day and the other component observed at southern latitudes $(<$ $-10^{\circ}$ ) has a rotation rate of $\sim 800^{\circ} /$ day. The SKR apparently is modulated at different rates in the two hemispheres, which obviously is difficult to explain if plasma and magnetic fields responsible for the emission generation, are interconnected between the hemispheres. Asymmetries, however, are introduced via the difference in solar illumination. So it might not be surprising that the rotation rates of both components cross each other, perhaps with some delay time after equinox (Fig. 4). A detailed discussion in Gurnett et al. (2009) further outlines the possibilities and constraints.

\section{Exoplanetary radio emission}

One of the first suggestions regarding the possibility of radio emission of exoplanets was provided by Farrell et al. (1999). Exoplanetary radio emission has not yet been found and there are various reasons for the non-detection. Among these are the beamed nature of the cyclotron maser instability, the insufficient sensitivity of the instruments, the mismatch of the transmitted and received frequencies, or that the radio emission frequency lies below the ionospheric cutoff (Vidotto et al., 2012). Many searches for exoplanetary radio emission have been made with the existing ground-based facilities. One of the more recent attempts was performed by Lecavelier des Etangs et al. (2013), who found a hint of radio emission around $150 \mathrm{MHz}$ from a Neptune-mass extrasolar planet named HAT-P-11b. Zarka et al. (2012) propose building a radio array on the moon to overcome the problem of the ionospheric cutoff. The emphasis, however, is the detection of exoplanetary radio emission from the terrestrial surface. Hallinan et al. (2013) describe an unsuccessful attempt to detect radio emission at $150 \mathrm{MHz}$ from the extrasolar planet $\tau$ Bootis $\mathbf{b}$ with the GMRT (Giant Meterwave Radio Telescope). Additional searches have been reported by Bastian et al. (2000) using the VLA (Very Large Array), as well as George and Stevens (2007) using the GMRT. The currently most sensitive radio array at decametric frequencies is the UTR-2 (Konovalenko et al., 2001) in Kharkiv (Ukraine), operating from 8 to $32 \mathrm{MHz}$ with an effective area of $150000 \mathrm{~m}^{2}$. An extensive observation campaign was 


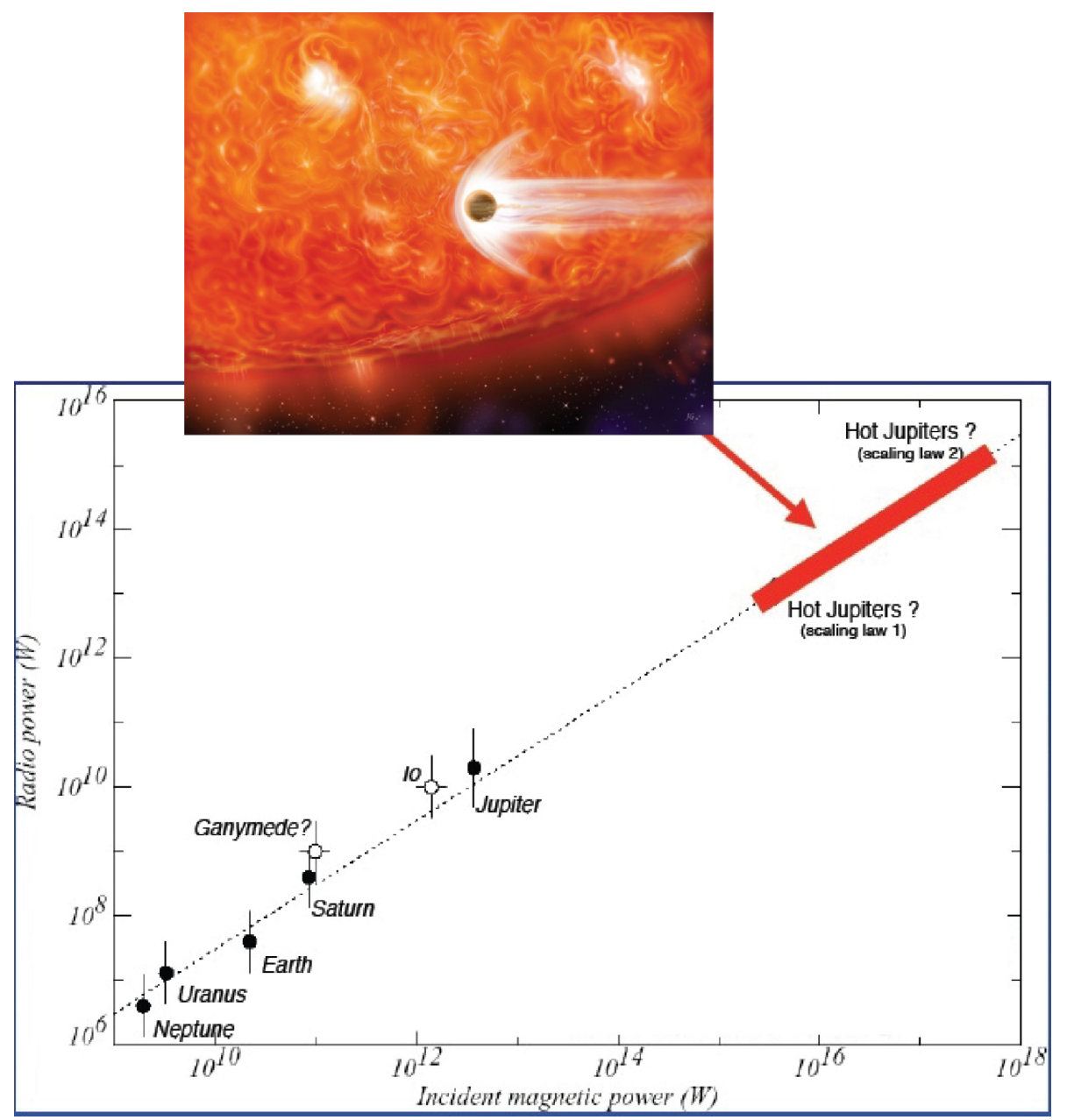

Figure 5. Expected radio flux of Hot Jupiters in comparison to solar system bodies (after Zarka, 2004).

carried out between 1997 and 2001 (Ryabov et al., 2004), but also without positive detection of exoplanetary radio emission.

Predictions for the detectability of exoplanetary radio emission from the ground have been made. Most of these statistics use the so-called radiometric Bode's law (empirical relation between incident solar wind power, magnetic field strength of the planet and the mean emitted radio power) to derive an estimation for the magnetic moment of the exoplanet (Lazio et al., 2004; Grießmeier et al., 2007; Grießmeier, 2007; Zarka et al., 2001). Nichols $(2011,2012)$ considers the configuration of an exoplanet with a satellite like Io orbiting the exoplanet. As is well known from the solar system planet Jupiter, the plasma interaction with its satellite Io produces strong radio emission (up to $40 \mathrm{MHz}$ ). All these predictions lead to the hypothesis that exoplanetary radio emission should be at or at least near the detection limit of current and future radio arrays, especially the UTR-2 and the LOFAR (LOw Frequency ARray) radio telescopes. The estimated radio flux is predicted to be $10^{4}$ or even $10^{5}$ times higher than for Jupiter in the solar system (Fig. 5). Hot Jupiters (Jovian mass planets at about 0.045 AU orbit around their host stars) and Jupiter-like planets with an inner plasma source like Io is for Jupiter (Nichols, 2011, 2012), are most likely to be detectable because the stellar wind kinetic energy input into the magnetosphere of such a planet is much larger compared to planets at larger orbital distances. Especially for younger and thus more active stars one can also expect stellar coronal mass ejections frequently hitting the planet (Grießmeier et al., 2007) and thus deposit much more energy into the magnetosphere and ultimately lead to higher radio fluxes. A tidally locked planet, due to slower rotation rate and thus smaller magnetic moment, would have a much smaller magnetosphere and thus also a smaller radio flux output. The influence of tidal locking on the magnetosphere of an exoplanet has been considered by Grießmeier et al. (2004). Lazio et al. (2004) were the first to present a full list of predictions for radio fluxes of all exoplanets known at that time derived from a radiometric Bode law. This list was updated by Grießmeier et al. (2007). Reiners and Christensen 
(2010) present a magnetic field evolution scenario for brown dwarfs and magnetic planets and from this model they derive an updated list of possibly detectable exoplanets. They base their study on dynamo scaling laws rather than a radiometric Bode's law.

The importance of detecting exoplanetary radio emission is the information this provides about exoplanetary magnetospheres. The maximum frequency of the emission provides information about the magnetic field strength of the planet. Also the orbital parameters or general information about the plasma environment of an exoplanet could be derived. Hess and Zarka (2011) show simulated exoplanet radio emission spectra and discuss in detail which features can be derived from spectra potentially measured in the future by UTR- 2 or LOFAR.

\section{Conclusions}

Non-thermal planetary radio emission is a valuable indicator for characteristic planetary and magnetospheric phenomena. Specific conditions in a magnetoplasma transform particle kinetic energy into electromagnetic wave energy where beams of keV electrons get into loss cone or shell distribution with subsequent emission of radio waves into thin walls of hollow emission cones. This hollow cone geometry is a general feature of planetary radiation, even observed in the Uranus kilometric radiation UKR when Voyager 2 observed the "bite-out" features looking into the hollow cone void of radio emission when the cone is swept over the spacecraft due to planetary rotation.

Planetary radio emission is modulated by planetary rotation and by external forces such as planetary satellites (Io in the case of Jupiter, possibly Rhea and Dione in the case of Saturn) and above all by the solar wind.

A most intriguing puzzle appeared at Saturn when Cassini RPWS observed the dual rotation rate from the Northern and Southern hemispheres, respectively. The underlying physical chain of processes is still matter of discussion.

Planetary radio emission is valuable for remote sensing of auroral and magnetospheric plasma dynamics and fields, and thus of considerable importance for its potential application to exoplanets. A first positive detection of exoplanetary radio emission may occur in the near future. Following up on this discovery, further parameters of the exoplanet, such as its magnetic environment and potentially the interaction with its host star via stellar winds, can be derived.
Acknowledgements. The authors are very thankful to the referees for their valuable and constructive comments and suggestions. The work of M. Panchenko was supported by the Austrian Fonds zur Förderung der wissenschaftlichen Forschung (project P23762N16). The work of C. Weber was supported by an OELZELT stipend of the Austrian Academy of Sciences.

Edited by: A. Kraus

Reviewed by: two anonymous referees

\section{References}

Anderson, J. D. and Schubert, G.: Saturn's Gravitational Field, Internal Rotation, and Interior Structure, Science, 317, 1384, doi:10.1126/science.1144835, 2007.

Bastian, T. S., Dulk, G. A., and Leblanc, Y.: A Search for Radio Emission from Extrasolar Planets, Astrophys. J., 545, 10581063, doi:10.1086/317864, 2000.

Benediktov, Y. A., Getmantsev, G. G., Mityakov, N. A., Rapoport, V. O., Sazonov, Y. A., and Tarasov, A. F.: Results of Radio Emission Strength Measurements at 725 and $1525 \mathrm{kc}$ by Equipment on the Satellite "Elektron-2", in: Space Research: Transactions of the All-union Conference on Space Physics, edited by: Skuridin, G. A., Al'pert, V. a. L., Krasovskiy, V. I., and Shvarev, V. V., 776-805, 1966.

Burke, B. F. and Franklin, K. L.: Observations of a Variable Radio Source Associated with the Planet Jupiter, J. Geophys. Res., 60, 213-217, doi:10.1029/JZ060i002p00213, 1955.

Carr, T. D., Desch, M. D., and Alexander, J. K.: Phenomenology of magnetospheric radio emissions, in: Physics of the Jovian Magnetosphere, edited by: Dessler, A. J., Cambridge Univ. Press, New York, pp. 226-284, 1983.

Cecconi, B. and Zarka, P.: Model of a variable radio period for Saturn, J. Geophys. Res., 110, 12203, doi:10.1029/2005JA011085, 2005.

Desch, M. D.: Evidence for solar wind control of Saturn radio emission, J. Geophys. Res., 87, 4549-4554, 1982.

Ergun, R. E., Carlson, C. W., McFadden, J. P., Mozer, F. S., Delory, G. T., Peria, W., Chaston, C. C., Temerin, M., Elphic, R., Strangeway, R., Pfaff, R., Cattell, C. A., Klumpar, D., Shelly, E., Peterson, W., Moebius, E., and Kistler, L.: FAST satellite wave observations in the AKR source region, Geophys. Res. Lett., 25, 2061-2064, 1998.

Ergun, R. E., Carlson, C. W., McFadden, J. P., Delory, G. T., Strangeway, R. J., and Pritchett, P. L.: Electron-Cyclotron Maser Driven by Charged-Particle Acceleration from Magnetic Fieldaligned Electric Fields, Astrophys. J., 538, 456-466, 2000.

Farrell, W. M., Desch, M. D., and Zarka, P.: On the possibility of coherent cyclotron emission from extrasolar planets, J. Geophys. Res., 104, 14025-14032, doi:10.1029/1998JE900050, 1999.

Farrell, W. M., Kaiser, M. L., Kurth, W. S., Desch, M. D., Gurnett, D. A., Hospodarsky, G. B., and MacDowall, R. J.: Remote sensing of possible plasma density bubbles in the inner Jovian dayside magnetosphere, J. Geophys. Res., 109, A09S14, doi:10.1029/2003JA010130, 2004.

Galopeau, P. H. M. and Lecacheux, A.: Variations of Saturn's radio rotation period measured at kilometer wavelengths, J. Geophys. Res., 105, 13089-13102, doi:10.1029/1999JA005089, 2000. 
George, S. J. and Stevens, I. R.: Giant Metrewave Radio Telescope low-frequency observations of extrasolar planetary systems, Mon. Not. R. Astron. Soc., 382, 455-460, doi:10.1111/j.13652966.2007.12387.x, 2007.

Grießmeier, J.-M.: Aspects of the magnetosphere stellar wind interaction of close-in extrasolar planets, Planet. Space Sci., 55, 530-531, doi:10.1016/j.pss.2006.11.003, 2007.

Grießmeier, J.-M., Stadelmann, A., Penz, T., Lammer, H., Selsis, F., Ribas, I., Guinan, E. F., Motschmann, U., Biernat, H. K., and Weiss, W. W.: The effect of tidal locking on the magnetospheric and atmospheric evolution of "Hot Jupiters", Astron. Astrophys., 425, 753-762, doi:10.1051/0004-6361:20035684, 2004.

Grießmeier, J.-M., Zarka, P., and Spreeuw, H.: Predicting lowfrequency radio fluxes of known extrasolar planets, Astron. Astrophys., 475, 359-368, doi:10.1051/0004-6361:20077397, 2007.

Gurnett, D. A., Kurth, W. S., Hospodarsky, G. B., Persoon, A. M., Averkamp, T. F., Cecconi, B., Lecacheux, A., Zarka, P., Canu, P., Cornilleau-Wehrlin, N., Galopeau, P., Roux, A., Harvey, C., Louarn, P., Bostrom, R., Gustafsson, G., Wahlund, J.-E., Desch, M. D., Farrell, W. M., Kaiser, M. L., Goetz, K., Kellogg, P. J., Fischer, G., Ladreiter, H.-P., Rucker, H., Alleyne, H., and Pedersen, A.: Radio and Plasma Wave Observations at Saturn from Cassini's Approach and First Orbit, Science, 307, 1255-1259, doi:10.1126/science.1105356, 2005.

Gurnett, D. A., Lecacheux, A., Kurth, W. S., Persoon, A. M., Groene, J. B., Lamy, L., Zarka, P., and Carbary, J. F.: Discovery of a north-south asymmetry in Saturn's radio rotation period, Geophys. Res. Lett., 36, 16102, doi:10.1029/2009GL039621, 2009

Gurnett, D. A., Groene, J. B., Persoon, A. M., Menietti, J. D., Ye, S.-Y., Kurth, W. S., MacDowall, R. J., and Lecacheux, A.: The reversal of the rotational modulation rates of the north and south components of Saturn kilometric radiation near equinox, Geophys. Res. Lett., 37, L24101, doi:10.1029/2010GL045796, 2010.

Hallinan, G., Sirothia, S. K., Antonova, A., Ishwara-Chandra, C. H., Bourke, S., Doyle, J. G., Hartman, J., and Golden, A.: Looking for a Pulse: A Search for Rotationally Modulated Radio Emission from the Hot Jupiter, $\tau$ Boötis b, Astrophys. J., 762, 34, doi:10.1088/0004-637X/762/1/34, 2013.

Hess, S. L. G. and Zarka, P.: Modeling the radio signature of the orbital parameters, rotation, and magnetic field of exoplanets, Astron. Astrophys., 531, A29, doi:10.1051/0004-6361/201116510, 2011.

Kaiser, M. L.: Time-variable magnetospheric radio emissions from Jupiter, J. Geophys. Res., 98, 18757, doi:10.1029/93JE01279, 1993.

Kaiser, M. L. and Desch, M. D.: Saturnian kilometric radiation - Source locations, J. Geophys. Res., 87, 4555-4559, doi:10.1029/JA087iA06p04555, 1982.

Kaiser, M. L. and MacDowall, R. J.: Jovian radio "bullseyes" observed by Ulysses, Geophys. Res. Lett., 25, 3113-3116, doi:10.1029/98GL02255, 1998.

Kaiser, M. L., Desch, M. D., Warwick, J. W., and Pearce, J. B.: Voyager detection of nonthermal radio emission from Saturn, Science, 209, 1238-1240, 1980.

Kaiser, M. L., Desch, M. D., and Lecacheux, A.: Saturnian kilometric radiation - Statistical properties and beam geometry, Nature, 292, 731-733, doi:10.1038/292731a0, 1981.
Kaiser, M. L., Zarka, P., Kurth, W. S., Hospodarsky, G. B., and Gurnett, D. A.: Cassini and Wind stereoscopic observations of Jovian nonthermal radio emissions: Measurement of beam widths, J. Geophys. Res., 105, 16053-16062, doi:10.1029/1999JA000414, 2000.

Konovalenko, A. A., Lecacheux, A., Rosolen, C., and Rucker, H. O.: New Instrumentations and Methods for the Low Frequency Planetary Radio Astronomy, in: Planetary Radio Emissions V, edited by: Rucker, H. O., Kaiser, M. L., and Leblanc, Y., p. $63,2001$.

Kurth, W. S., Averkamp, T. F., Gurnett, D. A., Groene, J. B., and Lecacheux, A.: An update to a Saturnian longitude system based on kilometric radio emissions, J. Geophys. Res., 113, A05222, doi:10.1029/2007JA012861, 2008.

Lamy, L.: Variability of southern and northern periodicities of Saturn Kilometric Radiation, in: Planetary Radio Emissions VII, edited by: Rucker, H. O., Kurth, W. S., Louarn, P., and Fischer, G., p. 38-50, 2011.

Lamy, L., Zarka, P., Cecconi, B., Prangé, R., Kurth, W. S., and Gurnett, D. A.: Saturn kilometric radiation: Average and statistical properties, J. Geophys. Res., 113, A07201, doi:10.1029/2007JA012900, 2008.

Lazio, W. T. J., Farrell, W. M., Dietrick, J., Greenlees, E., Hogan, E., Jones, C., and Hennig, L. A.: The Radiometric Bode's Law and Extrasolar Planets, Astrophys. J., 612, 511-518, doi:10.1086/422449, 2004.

Leblanc, Y., Genova, F., and de La Noe, J.: The Jovian S-bursts. I - Occurrence with L-bursts and frequency limit, Astron. Astrophys., 86, 342-348, 1980.

Lecavelier des Etangs, A., Sirothia, S. K., Gopal-Krishna, and Zarka, P.: Hint of $150 \mathrm{MHz}$ radio emission from the Neptunemass extrasolar transiting planet HAT-P-11b, Astron. Astrophys., 552, A65, doi:10.1051/0004-6361/201219789, 2013.

Louarn, P., Roux, A., de Feraudy, H., Le Queau, D., and Andre, M.: Trapped electrons as a free energy source for the auroral kilometric radiation, J. Geophys. Res., 95, 5983-5995, 1990.

Nichols, J. D.: Magnetosphere-ionosphere coupling at Jupiter-like exoplanets with internal plasma sources: implications for detectability of auroral radio emissions, Mon. Not. R. Astron. Soc., 414, 2125-2138, doi:10.1111/j.1365-2966.2011.18528.x, 2011.

Nichols, J. D.: Candidates for detecting exoplanetary radio emissions generated by magnetosphere-ionosphere coupling, Mon. Not. R. Astron. Soc., 427, L75-L79, doi:10.1111/j.17453933.2012.01348.x, 2012.

Panchenko, M. and Rucker, H. O.: New Type of Periodic Bursts of Non-Io Jovian Decametric Radio Emission, in: Planetary Radio Emissions VII, edited by: Rucker, H. O., Kurth, W. S., Louarn, P., and Fischer, G., 157-166, 2011.

Panchenko, M., Rucker, H. O., Kaiser, M. L., St. Cyr, O. C., Bougeret, J., Goetz, K., and Bale, S. D.: New periodicity in Jovian decametric radio emission, Geophys. Res. Lett., 37, 5106, doi:10.1029/2010GL042488, 2010.

Panchenko, M., Rucker, H. O., and Farrell, W. M.: Periodic bursts of Jovian non-Io decametric radio emission, Planet. Space Sci., 77, 3-11, doi:10.1016/j.pss.2012.08.015, 2013.

Read, P. L., Dowling, T. E., and Schubert, G.: Saturn's rotation period from its atmospheric planetary-wave configuration, Nature, 460, 608-610, doi:10.1038/nature08194, 2009. 
Reiners, A. and Christensen, U. R.: A magnetic field evolution scenario for brown dwarfs and giant planets, Astron. Astrophys., 522, A13, doi:10.1051/0004-6361/201014251, 2010.

Riihimaa, J. J.: Modulation Lanes in the Dynamic Spectra of Jovian L Bursts, Astron. Astrophys., 4, 180-188, 1970.

Riihimaa, J. J.: S-bursts in Jupiter's decametric radio spectra, Astrophys. Space Sci., 51, 363-383, doi:10.1007/BF00644159, 1977.

Ryabov, V. B., Zarka, P., and Ryabov, B. P.: Search of exoplanetary radio signals in the presence of strong interference: enhancing sensitivity by data accumulation, Planet. Space Sci., 52, 14791491, doi:10.1016/j.pss.2004.09.019, 2004.

Sandel, B. R. and Dessler, A. J.: Dual periodicity of the Jovian magnetosphere, J. Geophys. Res., 93, 5487-5504, doi:10.1029/JA093iA06p05487, 1988.

Saur, J., Neubauer, F. M., Connerney, J. E. P., Zarka, P., and Kivelson, M. G.: Plasma interaction of Io with its plasma torus, in: Jupiter. The Planet, Satellites and Magnetosphere, edited by: Bagenal, F., Dowling, T. E., and McKinnon, W. B., Cambridge University Press, 537-560, 2004.

Steffl, A. J., Delamere, P. A., and Bagenal, F.: Cassini UVIS observations of the Io plasma torus. III. Observations of temporal and azimuthal variability, Icarus, 180, 124-140, doi:10.1016/j.icarus.2005.07.013, 2006.

Treumann, R. A.: The electron-cyclotron maser for astrophysical application, Astron. Astrophys. Rev., 13, 229-315, doi:10.1007/s00159-006-0001-y, 2006.
Vidotto, A. A., Fares, R., Jardine, M., Donati, J.-F., Opher, M., Moutou, C., Catala, C., and Gombosi, T. I.: The stellar wind cycles and planetary radio emission of the $\tau$ Boo system, Mon. Not. R. Astron. Soc., 423, 3285-3298, doi:10.1111/j.13652966.2012.21122.x, 2012.

Wu, C. S.: Kinetic cyclotron and synchrotron maser instabilities Radio emission processes by direct amplification of radiation, Space Sci. Rev., 41, 215-298, doi:10.1007/BF00190653, 1985.

$\mathrm{Wu}, \mathrm{C}$. S. and Lee, L. C.: A theory of the terrestrial kilometric radiation, Astrophys. J., 230, 621-626, 1979.

Zarka, P.: Auroral radio emissions at the outer planets: Observations and theories, J. Geophys. Res., 103, 20159-20194, doi:10.1029/98JE01323, 1998.

Zarka, P.: Non-thermal radio emissions from extrasolar planets, in: Extrasolar Planets: Today and Tomorrow, 321, 160-169, 2004.

Zarka, P., Treumann, R. A., Ryabov, B. P., and Ryabov, V. B.: Magnetically-Driven Planetary Radio Emissions and Application to Extrasolar Planets, Astrophys. Space Sci., 277, 293-300, doi:10.1023/A:1012221527425, 2001.

Zarka, P., Bougeret, J.-L., Briand, C., Cecconi, B., Falcke, H., Girard, J., Grießmeier, J.-M., Hess, S., Klein-Wolt, M., Konovalenko, A., Lamy, L., Mimoun, D., and Aminaei, A.: Planetary and exoplanetary low frequency radio observations from the Moon, Planet. Space Sci., 74, 156-166, doi:10.1016/j.pss.2012.08.004, 2012.

Zörweg, P.: Multi-spacecraft data analysis of Jovian radio emission, Diplomarbeit, 66 p., Karl-Franzens University Graz, Austria, 2011. 